\title{
Life expectancy grows with supply of primary care doctors
}

\author{
— Cite as: CMAJ 2019 March 25;191:E347. doi: 10.1503/cmaj.109-5729
}

Posted on cmajnews.com on Mar. 6, 2019.

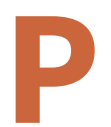

eople live longer in areas with more primary care doctors, according to data from the United States. But the supply of these doctors is shrinking as more medical students choose higher paying specialties.

Life expectancy increased 51.5 days for every 10 more primary care doctors per 100000 people in the United States between 2005 and 2015, according to a study led by researchers at Stanford and Harvard universities. A similar increase in other specialists boosted life expectancy only 19.2 days.

Areas with more primary care doctors also had lower mortality rates. There were $0.9 \%$ fewer cardiovascular deaths, $1 \%$ fewer cancer deaths, and $1.4 \%$ fewer respiratory deaths for every 10 more primary care doctors per 100000 people.

"Greater supply of primary care physicians appeared to increase the chances that a person would be treated for cardiovascular disease risk factors like high blood pressure or high cholesterol, or caught early for major cancers like breast cancer or colon cancer," according to lead author Dr. Sanjay Basu.

Basu's team examined data from 3142 counties, 7144 primary care service areas and 306 hospital referral regions. Unlike some previous studies, they accounted for regional factors that might influence longevity, such as poverty levels and numbers of hospital beds, as well as individual factors, including smoking habits and obesity.

The researchers also looked at people who moved between areas with different densities of primary care physicians and compared how their survival changed, controlling for regional and individual characteristics. They found that people who moved to areas with more primary care doctors increased their life expectancy by as

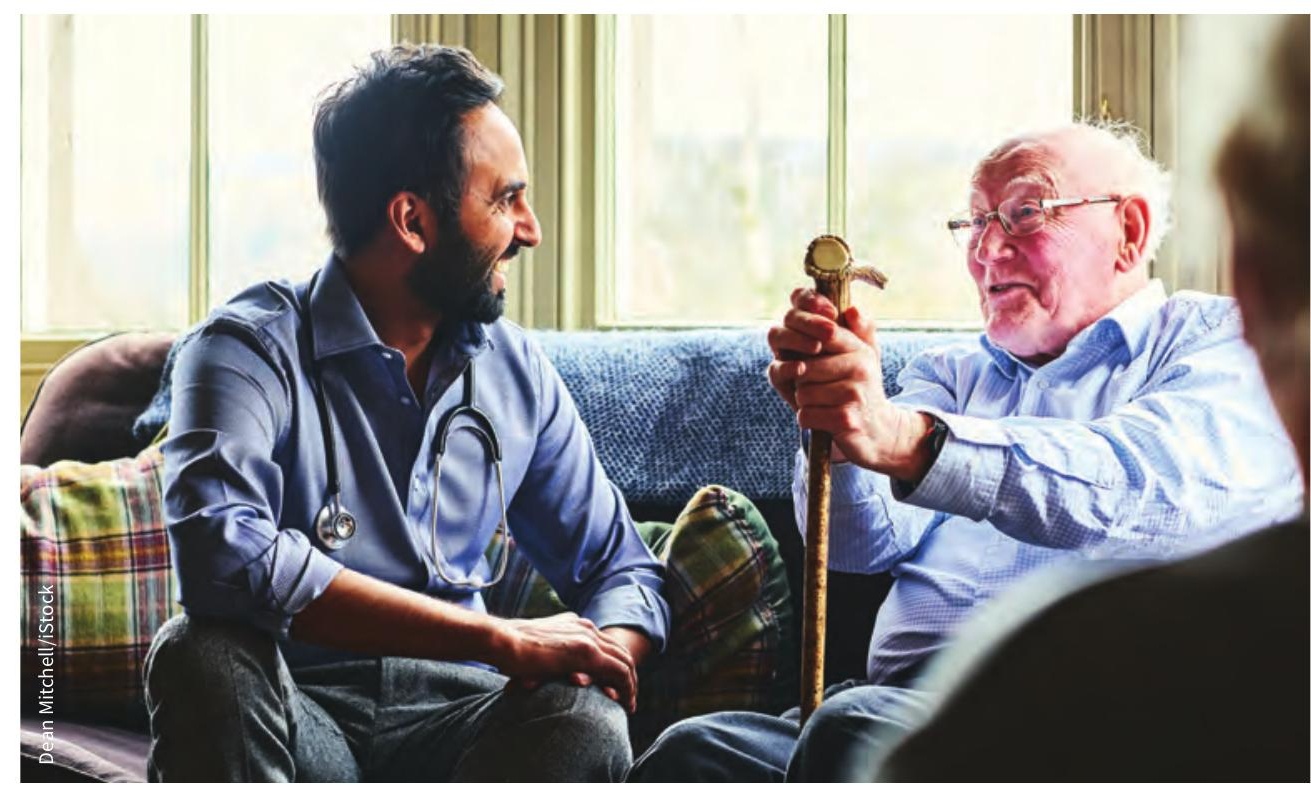

Better access to primary care may boost longevity, a new study finds.

much as 114.2 days per decade for every 10 additional physicians per 100000 people.

Despite the correlation between primary care and longer lives, access is worsening, the study authors warned. Although the total number of primary care doctors in the US has increased in recent years, it hasn't kept pace with overall population growth or the exodus of providers from rural areas. Taking these trends into account, the number of primary care doctors per 100000 people dropped from 46.6 to 41.4 between 2005 and 2015. The Association of American Medical Colleges predicts a major shortfall of 14800 to 49300 primary care doctors by 2030 .

According to Basu, there are few incentives for medical graduates to go into primary care. "Pay tends to be lower, burnout rates higher and prestige lower."

Similar pressures are contributing to family doctor shortages and long waits for primary care in Canada. Although the total number of doctors in Canada has grown at rates faster than the population for more than a decade, family medicine has struggled to attract physicians to its ranks. Meanwhile, increasing numbers of family doctors are working as hospitalists or narrowing their practices to niche interests.

Money is a factor in these trends. Average payments to family doctors have decreased in recent years while those to other specialists have increased. Family doctors working as hospitalists pay less overhead than those working in the community.

In a commentary accompanying the Stanford study, Sondra Zabar and colleagues call for financial incentives to attract more doctors into primary care and underserved areas. "Physician payment reform is a key to making all of this happen."

\section{Lauren Vogel, CMAJ}

